\title{
Newly classified horizontal canal positional nystagmus and its treatment
}

\author{
Kiyoshi Hiruma $^{1 *}$, Akihiro Ohara ${ }^{2}$ and Izumi Koizuka ${ }^{2}$ \\ ${ }^{1}$ Department of Otorhinolaryngology, Kawasaki Municipal Tama Hospital, Kawasaki, Japan \\ ${ }^{2}$ Department of Otorhinolaryngology, St. Marianna University School of Medicine, Kawasaki, Japan
}

\begin{abstract}
Objectives: Horizontal canal type BPPV shows various types of direction-changing positional nystagmus (DCPN). The aim of this study was to classify into 4 types by the duration (persistent without latency or transient with latency) and the direction (apogeotropic or geotropic) and describe the details of each type and to discuss each pathophysiological mechanism and treatment.
\end{abstract}

Study design and setting: Retrospective case series

Participants: We conducted a retrospective case series study in 50 patients with DCPN. Positional nystagmus was examined using an infrared camera or Frenzel glasses.

Main outcome measures: We compared clinical outcome parameters (frequency, affected side, sex, treatment) among the 4 types.

Results: 1) persistent apogeoropic type (22\%), right side(R): left side(L)=10:1, Male(M): Female(F)=6:5, Yaw head rolling or conservative therapy, 2) persistent geotropic type (20\%), $R: L=5: 5, M: F=4: 6$, conservative therapy, 3) transient apogeotropic (10\%), $R: L=4: 1, M: F=2: 3$, Yaw head rolling, 4) transient geotropic type (48\%), R:L=14:10, M:F=14:10, Barbecue rotation or Forced Prolonged Position (FPP).

Conclusion: Each pathophysiology was thought to be that 1) persistent apogeoropic type was heavy cupula including cupulolithiasis, 2) persistent geotropic type was light cupula, 3) transient apogeotropic was canalolithiasis with the debris within the short arm side of the canal, 4) transient geotropic type was canalolithiasis with the debris within the long arm side of the canal.

\section{Introduction}

The most common type of benign paroxysmal positional vertigo (BPPV) is posterior semicircular canal BPPV, which is characterized by rotatory nystagmus on the Dix-Hallpike maneuver. On the contrary, direction-changing positional nystagmus (DCPN) is well reported as the horizontal semicircular canal type BPPV recently. Horizontal semicircular canal positional vertigo showing DCPN is a relatively new disease concept and no consensus has been reached concerning its pathophysiology or optimal treatment. So, we proposed new classification of horizontal semicircular canal positional nystagmus and analyzed DCPN cases retrospectively.

We classified horizontal canal positional vertigo into persistent type and transient type. In persistent type, nystagmus lasts more than one minute and the nystagmus, while in transient type the nystagmus decays and stopped within 30 seconds. Additionally, DCPN is classified into apogeotropic and geotropic positional nystagmus. So, four types are classified into 1) persistent apogeotropic nystagmus, 2) persistent geotropic nystagmus, 3) transient apogeotropic nystagmus, 4) transient geotropic nystagmus.

The purpose of this study was to describe the four types of horizontal semicircular canal positional nystagmus in detail and discuss their pathophysiological mechanisms and treatment for each.

\section{Materials and methods}

We conducted a retrospective study of 50 patients (26 males, 24 females; mean age, 66.4 years; age range, $30-94$ years) with DCPN due to horizontal canal who were treated at Tokyo Metropolitan Komagome Hospital between October 2005 and January 2014. Positional nystagmus was examined using an infrared camera installed in goggles (Infrared Frenzel Goggles, DAIICHI MEDICAL CO., LTD, Tokyo, Japan) or Frenzel glasses. No patients had nystagmus when in a sitting position. No patients had any central nervous system disorder.

\section{Nystagmus testing}

To examine persistent positional nystagmus, patients were turned in the axial plane very slowly so as not to induce dynamic positional nystagmus. Patients with persistent nystagmus (right or left beating) without latency in the supine position were categorized as persistent DCPN. This positional nystagmus ceases when the head is turned from the supine position to the one side (we call the first neural point). The first neutral point was identified by rotating the head 20-30 degrees to the right or left and was the head position at which both vertigo and nystagmus disappeared (Figure 1). The second neutral point was 180

${ }^{\star}$ Correspondence to: Kiyoshi Hiruma, Department of Otorhinolaryngology, Kawasaki Municipal Tama Hospital, 1-30-37, Shukugawara, Tama, Kawasaki, 214-8525, Japan, Tel: +81-44-933-8111; Fax: 81-44-930-5181; E-mail: ZBN22654@nifty.com

Key words: direction-changing positional nystagmus, cupulolithiasis, canalolithiasis, heavy cupula, light cupula, short arm, long arm

Received: October 13, 2018; Accepted: October 19, 2018; Published: October 26, 2018 


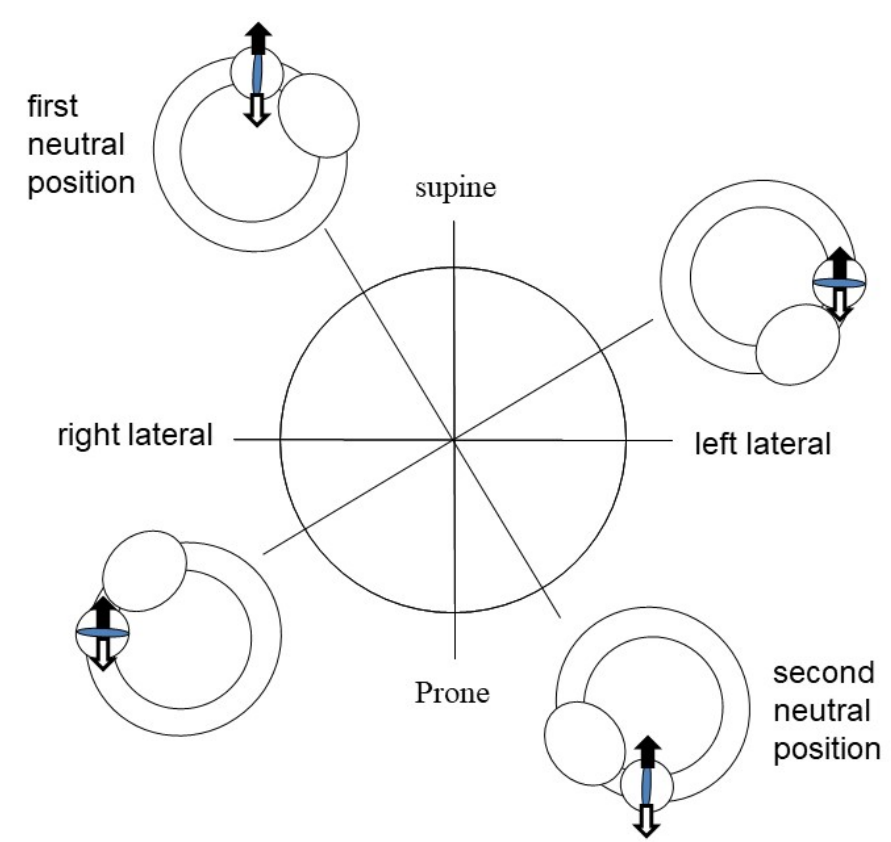

Figure 1. Schema showing the cupula of the right horizontal semicircular canal

The two neutral points are 180 degrees away from each other. At the each neutral point, the cupula is positioned vertical to the gravitational plane, and there is no deflection of the cupula. When the head is tilted at 90 degrees yaw from the neutral points, the cupula is positioned horizontal to the gravitational plane, and both vertigo and nystagmus become intense. If the cupula is heavier than the surrounding endolymph in the horizontal canal, it acts as "heavy cupula" and is pulled downward maximally (white arrow), resulting in persistent apogeotropic DCPN. If the cupula is lighter than the surrounding endolymph in the horizontal canal, it acts as "light cupula" and is pushed upward maximally (black arrow), resulting in persistent geotropic DCPN

degrees from the first neutral point (Figure 1). Positional nystagmus persisted in all positions except the two neutral points. Patients with nystagmus beating toward the first neutral point were categorized as persistent apogeotropic type DCPN and patients with nystagmus beating away from the first neutral point were categorized as persistent geotropic type DCPN [1-3]. The side on which the first neutral point was located was determined as the affected side $[2,3]$.

Patients without nystagmus in the supine position but with beating nystagmus with latency that decayed within $30 \mathrm{~s}$ when rolled onto the right side (right lateral position) and left side (left lateral position) were categorized as transient DCPN. Patients with left-beating nystagmus in the right lateral position and right-beating nystagmus in the left lateral position were categorized as transient apogeotropic type DCPN [4] and patients with right-beating nystagmus in the right lateral position and left-beating nystagmus in the left lateral position were categorized as transient geotropic type DCPN. In patients with transient geotropic DCPN, nystagmus provoke more intense when the affected ear was lowermost and in patients with transient apogeotropic DCPN, nystagmus provoke more intense when the affected ear was uppermost [5].

DCPN type was determined at the time of the first visit to the hospital. If the nystagmus does not disappear within 2 weeks, newly treatment will be considered.

\section{Results}

Eleven patients (22\%; 6 males, 5 females) had persistent apogeotropic type DCPN, 10 patients (20\%; 4 males, 6 females) had persistent geotropic type DCPN, 5 patients (10\%; 2 males, 3 females) had transient apogeotropic type DCPN and 24 patients (48\%; 14 males, 10 females) had transient geotropic type DCPN (Figure 2).

\section{Persistent apogeotropic type DCPN (PA)}

All 11 patients with persistent apogeotropic type DCPN had a neutral point at which nystagmus ceased. The neutral point was 5-29 degrees from the supine position (average, 18.7 degrees; standard deviation, 8.1 degrees). Ten patients were affected on the right side and one patient was affected on the left side. With yaw rotation (head shaking), 3 patients changed to ipsilateral transient geotropic type DCPN and barbecue rotation (Lempert maneuver) and forced prolonged position (FPP) were effective at stopping nystagmus. With yaw rotation (yaw head shaking), 1 patient changed to ipsilateral posterior canal type BPPV and Epley's maneuver was effective. In the other 7 patients, nystagmus naturally disappeared after conservative treatment and did not convert to another type of nystagmus (Figure 3).

\section{4 types of positional nystagmus}

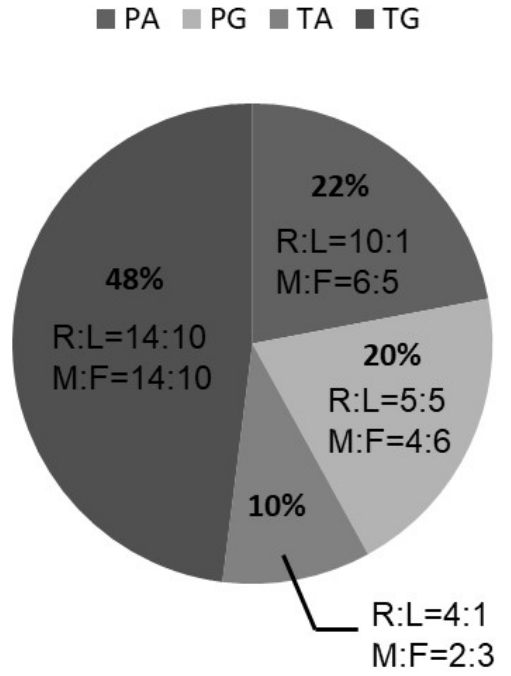

Figure 2. The gender and affected side of patients with each type of DCPN PA: persistent apogeotropic DCPN, PG: persistent geotropic DCPN, TA: transient apogeotropic DCPN, TG: transient geotropic DCPN, R: right side, L: left side,

M: Male, F: Female

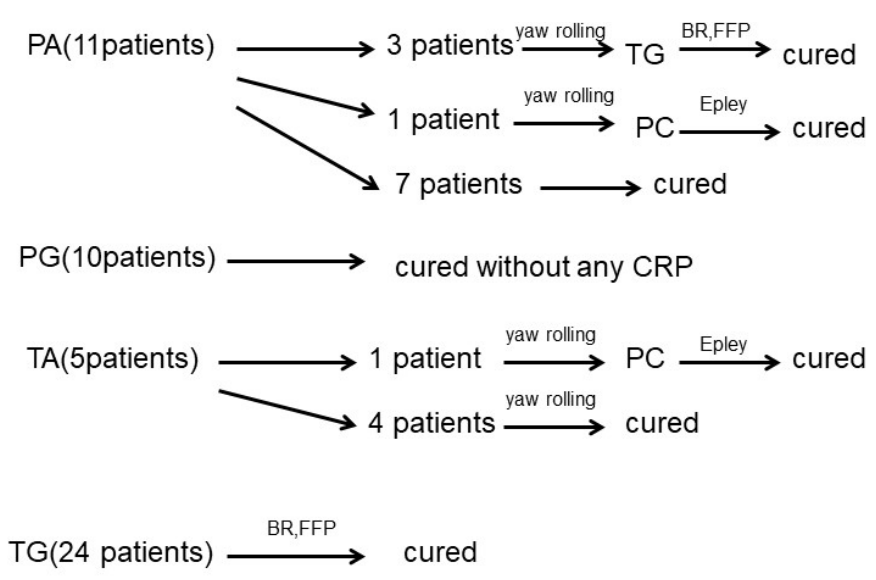

Figure 3. Clinical course of the 50 patients with DCPN included in this study

PA: persistent apogeotropic DCPN, PG: persistent geotropic DCPN, TA: transient apogeotropic DCPN, TG: transient geotropic DCPN, PC: posterior semicircular canal BPPV, FPP: forced prolonged position, CRP: canalith repositioning procedure 


\section{Persistent geotropic type DCPN (PG)}

All 10 patients with persistent geotropic type DCPN had a neutral point at which nystagmus ceased. The neutral point was 15-58 degrees from the supine position (average, 25.4 degrees; standard deviation, 12.5 degrees). Five patients were affected on the right side and 5 patients were affected on the left side. Prognosis of this type of DCPN was good without any canalith repositioning maneuver. (Figure 3).

The angle between the supine position and first neutral point showed no statistically significant difference between PA and PG (Mann-Whitney test).

\section{Transient apogeotropic type DCPN (TA)}

Four of the 5 patients with transient apogeotropic type DCPN were affected on the right side and one was affected on the left side. One patient changed to ipsilateral posterior canal type BPPV after yaw rotation and cured by Epley's maneuver. The other four patients did not change to transient geotropic type DCPN and cured by yaw rotation (Figure 3).

\section{Transient geotropic type DCPN (TG)}

Fourteen of the 24 patients with transient geotropic type DCPN were affected on the right side and 10 were affected on the left side. Barbecue rotation (Lempert maneuver) and FPP were effective in all 24 patients (Figure 3).

\section{Discussion}

Our patients with DCPN were able to be classified into four groups according to the duration (persistent without latency or transient with latency) and direction (apogeotropic or geotropic) of the nystagmus. 4 DCPN groups consist of 1) persistent apogeotropic, 2) persistent geotropic, 3) transient apogeotropic, 4) transient geotropic positional nystagmus. Each group had their pathophysiological characteristics and different treatment was needed for each group.

Persistent apogeotropic type DCPN is thought to be due to cupulolithiasis in the unilateral horizontal semicircular canal (HSCC) [6-8]. During yaw rotation, 3 patients with persistent apogeotropic type DCPN changed to transient geotropic type DCPN and 1 patient changed to ipsilateral posterior canal BPPV. Barbecue rotation (Lempert maneuver) and FPP were effective in the three patients that converted to transient geotropic type DCPN, suggesting that debris or otoconia that was attached to the long-arm-side of the cupula detached into the distal long arm of the HSCC during rotation to cause canalolithiasis that resulted in transient geotropic type DCPN (Figure 4). Epley's maneuver was effective in the patient that converted to ipsilateral posterior canal type BPPV, suggesting that debris or otoconia that was attached to the utricular side of the cupula detached into the proximal short arm during rotation and moved to the posterior SCC via the utricle to cause canalolithiasis that resulted in posterior canal BPPV [9] (Figure 4). Almost all patients with persistent apogeotropic type DCPN were affected on the right side, suggesting that this type of nystagmus may have been caused by otoconia from the utricle (otolithic) as posterior canal type BPPV [10]. The predominant involvement of the right side in posterior type BPPV may be due to the fact that most patients sleep on their right side and sleeping on the right side may facilitate entry of heavy particles from the utricle into the horizontal canal [10]. However, in seven of the 11 patients with persistent apogeotropic type DCPN the nystagmus disappeared after conservative treatment and did not convert to another type, suggesting

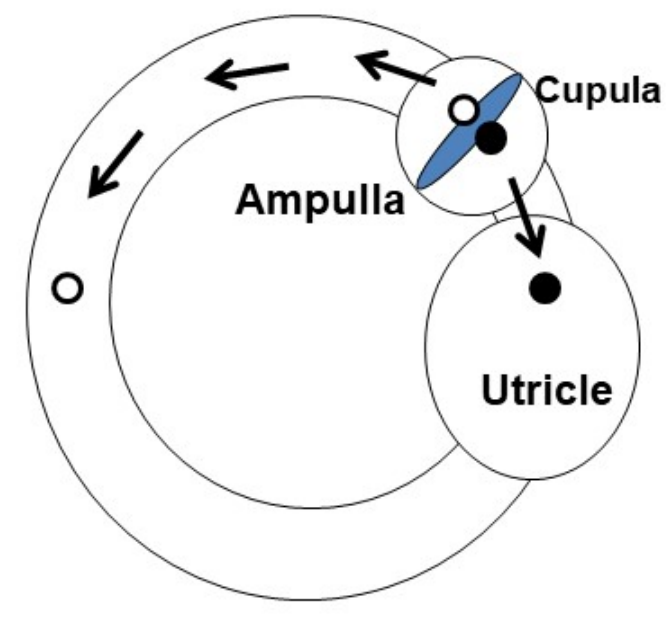

Figure 4. Trajectory of the otoconia from the long arm and short arm of the horizontal semicircular canal

The white circle represents long-arm side otoconia and the black circle represents short-arm side otoconia. The former may detach from the cupula into the distal long arm of the HSCC and the latter may detach from cupula into utricle via the short arm of the HSCC and move into posterior semicircular canal.

HSCC: horizontal semicircular canal

that it may have been caused by a cupula denser than the endolymph. This implies a condition of differential density between the cupula and the endolymph [7].

Persistent geotropic type DCPN is thought to be the mirror image of DCPN caused by heavy cupula, but its pathophysiology is different from the heavy cupula type and thought to be controversial "light cupula" due to the specific gravity of the endolymph in the unilateral HSCC [1-3], and not due to otoconia. The prognosis of patients with persistent geotropic type DCPN was good without any canalith repositioning maneuver, suggesting that the pathophysiology of persistent geotropic type DCPN may not be otolithic. However, the conversion to posterior canal type BPPV from persistent geotropic type DCPN (unpublished data) may suggest the presence of light debris [3], as it could be broken into pieces, conglomerate again, and then change to the true otoconia (heavy dedris) during the transition between canals.

Transient apogeotropic type DCPN is thought to be due to canalolithiasis caused by debris within the anterior part of the unilateral HSCC [5]. Nuti, et al. [5] reported that horizontal canal BPPV with apogeotropic DCPN changed to geotropic DCPN. It is possible that the free-floating otoconia located in the anterior part of the HSCC moved to the posterior part of the HSCC immediately after the change in gravity (Figure 5A). In our patients, this apogeotropic DCPN did not convert to geotropic DCPN before the nystagmus disappeared. We speculate that the free-floating otoconia were located in the ampulla region on the utricle (vestibular) side [4], therefore, yaw rotation and FPP may be effective maneuvers (Figure 5B).

Transient geotropic type DCPN is thought to be due to canalolithiasis caused by debris within the posterior part of the unilateral HSCC. This was the most common type of DCPN present in our cohort, and barbecue rotation (Lempert maneuver) and FPP were effective in all patients. Intractable DCPN is thought to be caused by "cupulolithiasis", or "canalith jam" [11], and conversion to transient geotropic type DCPN from any other type of DCPN after the yaw rotation or other maneuvers can solve the problem. This type of DCPN should be treated according to Nuti's criteria for management [12]. 


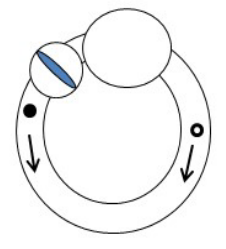

right lateral

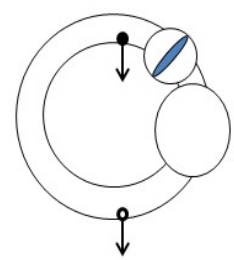

supine

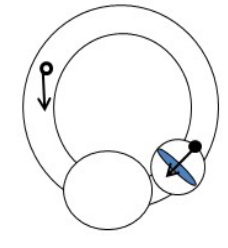

left lateral
Figure 5A. Canalolithiasis with the otoconia within the anterior and posterior part of the horizontal semicircular canal (right ear)

The black circle represents otoconia within the anterior part of the HSCC and induces transient apogeotropic DCPN, the white circle represents otoconia within the posterior part of the HSCC and induces transient geotropic DCPN

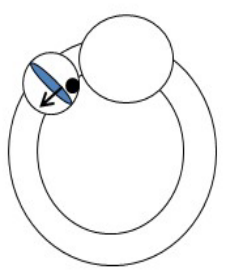

Right lateral

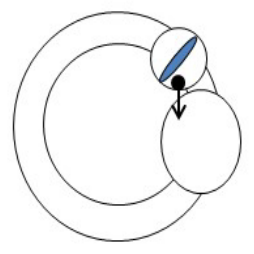

supine

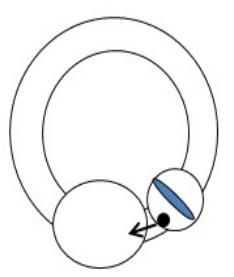

left lateral
Figure 5B. Canalolithiasis with the otoconia in the ampulla region on the utricle side (right ear) When the patient rolls onto their right side (right lateral position), the otoconia (black circle) moves towards the cupula, induces ampullofugal flow, and left beating, apogeotropic nystagmus is induced. In the supine position, the otoconia stays in the ampulla region on the utricle side and no nystagmus is produced. When the patient rolls onto their left side (left lateral position), the otoconia moves away from cupula, induces ampullopetal flow, and strong right beating, apogeotropic nystagmus is produced

\section{Pathophysiology of DCPN}

None of our patients had nystagmus in the sitting position. This means that the patients did not show spontaneous paralytic or irritative nystagmus. So, we do not consider the DCPN to be indicative of process in vestibular compensation. Positional nystagmus was evoked after specific positioning of the horizontal canal on its own plane. Three hypotheses should be considered for the pathogenesis of the four types of DCPN: 1) cupulolithiasis or canalolithiasis, 2) heavy cupula or light cupula, and 3) long-arm-side lesion or short-arm-side lesion.

Cupulolithiasis, or deposits on the cupula, can account for persistent nystagmus without latency. Canalolithiasis, a free-floating clot within the endolymph of canal, can account for transient nystagmus with latency. Though we did not have any patients that converted from canalolithiasis to cupulolithiasis in the course of horizontal BPPV, this has previously been reported [13,14]. Transient geotropic DCPN also transformed to persistent apogeotropic DCPN (our unpublished data). It can be assumed that otoconia debris adhered to the cupula and converted the canalolithiasis to cupulolithiasis of the HSCC on the same side. Conversion of apogeotropic nystagmus into geotropic lateral canalolithiasis during a head pitching maneuver in the sitting position has also been reported [15]. Transient geotropic DCPN (canalolithiasis) was effectively cured by the barbecue rotation [16]. Cupular deposits may be derived from the otolithic organ (maculae of the utricle and saccule), but the true origin remains uncertain [17] and local cupular lesions are possible.

Persistent apogeotropic DCPN can be explained by a heavy cupula that results from deposits (cupulolithiasis) or by a cupula that is denser than the endolymph [7]. Persistent geotropic DCPN can be explained by a light cupula that is less dense than in the surrounding endolymph $[1,2]$. Another explanation for light cupula is a reversible lesion such as a denatured cupula or utricular imbalance of the same ear [18]. In this study we determined the affected side as the side of the first neutral point, but the bow and lean test might have been helpful to confirm the affected side in some patients [19]. However, Kim reported that the side of the null plane, which corresponds to the first neutral point in this study, coincided with the affected side indicated by the bow and lean test, indicating that it is possible to determine the affected side from the side of the neutral point [20]. The neutral point is defined by the angle between the cupula and the gravity vector, but in our subjects, especially those with light cupula DCPN was deviated more than the expected true angle. The neutral point may therefore be affected by factors other than the anatomical position of the cupula [1], such as morphological changes. The alteration of specific gravity of the endolymph surrounding the cupula may cause heavy cupula and light cupula $[1,2,20]$, but this remains controversial.

To evaluate remission time and canal conversion, it is important to consider whether the lesion is on the long- or the short-arm side. Transient DCPN has previously been considered to be due to migration of free-floating debris from the ampulla to the utricle through the long arm of HSCC. However, the migration of free-floating debris from the ampulla of the utricular side to the utricle through the short arm of HSCC must also be considered [9,21]. The path from the ampulla to the utricle via the proximal short arm of HSCC is shorter than that via the distal long arm of the HSCC. Imai divided patients with apogeotropic nystagmus into the following two groups: the early remission group and the delayed remission group [9]. He reported that early remission group may be related to the movement of free-floating debris throughout the proximal short arm of HSCC, and the delayed remission group may be related to the distal long arm of the HSCC. One of our patients with transient apogeotropic type DCPN converted to ipsilateral posterior canal BPPV (canal conversion) without converting to transient geotropic DCPN. This indicates that debris moved in the short arm of HSCC [4]. Oas stated that utriculolithiasis must precede the development of positional vertigo, and that it is just a matter of time and positioning until the otoconia are displaced into the semicircular system [21]. Anatomically, the common crus of semicircular ducts receives the distal end of both the superior and posterior semicircular canals and five orifices of the three semicircular canals empty into the utricular sac. Oas also discussed the three semicircular canal orifices on the short-arm side and proposed that new terminology is required to differentiate short-arm from long-arm canalolithiasis [21].

In this study, we have described four types of DCPN and have proposed a treatment for each type (Table 1). The treatments were determined according to the proposed pathophysiology of the DCPN. It is very important to determine the type of DCPN at the first observation, especially in horizontal canal positional vertigo, because the nystagmus pattern can change rapidly. If the nystagmus pattern changes, the pathophysiology will change, as well the optimal treatment. Our classification may be useful in clinical practice when dealing with patients with DCPN, particularly those with nystagmus conversion or canal conversion.

\section{Conclusion}

There are different types of DCPN present in horizontal canal positional vertigo. We categorized the DCPN according to the direction (apogeotropic or geotropic) and duration (persistent without latency 
Table 1. Types and Treatment of DCPN

\begin{tabular}{|l|l|l|}
\hline \multicolumn{1}{|c|}{} & Pathophysiology & Treatment \\
\hline PA & $\begin{array}{l}\text { Cupulolithiasis (long arm side, short } \\
\text { arm side, both side) } \\
\text { Heavy cupula }\end{array}$ & Yaw head rolling $\rightarrow$ TG \\
\hline PG & Light cupula & Conservative therapy \\
\hline TA & $\begin{array}{l}\text { Canalolithiasis } \\
\text { Short arm side ampulla } \\
\text { Anterior part of long arm }\end{array}$ & $\begin{array}{l}\text { FPP (healthy side down) } \\
\text { Yaw head rolling } \rightarrow \text { TG }\end{array}$ \\
\hline TG & $\begin{array}{l}\text { Canalolithiasis } \\
\text { Posterior part of long arm }\end{array}$ & $\begin{array}{l}\text { Barbecue rotation (Lempert maneuver) } \\
\text { FPP (healthy side down) }\end{array}$ \\
\hline
\end{tabular}

or transient with latency). These categories of DCPN have different pathophysiologies and different treatments. The pathophysiology of the different types of DCPN in horizontal canal positional vertigo remains to be fully elucidated, but our classification may be useful in clinical practice.

\section{References}

1. Hiruma K, Numata T (2004) Positional nystagmus showing neutral points. ORL $J$ Otorhinolaryngol Relat Spec 66: 46-50. [Crossref]

2. Hiruma K, Numata T, Mitsuhashi T, Tomemori T, Watanabe R, et al. (2011) Two types of direction-changing positional nystagmus with neutral points. Auris Nasus Larynx 38: 46-51. [Crossref]

3. Ichijo H (2012) Persistent direction-changing geotropic vertigo positional nystagmus. Eur Arch Otorhinolaryngol 269: 747-751.

4. Hiruma K, Watanabe R (2013) Transient type of apogeotropic direction-changing positional nystagmus. Pract Otol 137: 6-7

5. Nuti D, Vannucchi P, Pagnini P (1996) Benign paroxysmal positional vertigo of horizontal canal: a form of canalolithiasis with variable clinical features. $J$ Vest Res 6: $173-184$

6. Bisdorff AR, Debatisse D (2001) Localizing signs in positional vertigo due to lateral canal cupulolithiasis. Neurology 57: 1085-1088.

7. Casani A, Giovanni V, Bruno F, Luigi GP (1997) Positional vertigo and apogeotropic bidirectional nystagmus. Laryngoscope 107: 807-813.
8. Ichijo H (2012) Cupulolithiasis of the horizontal semicircular canal. Eur Arch Otorhinolaryngol 269: 53-56.

9. Imai T, Takeda N, Ito M, Inohara H (2011) Natural course of positional vertigo in patients with apogeotropic variant of horizontal canal benign paroxysmal positional vertigo. Auris Nasus Larynx 38: 2-5. [Crossref]

10. von Brevern M, Seelig T, Neuhauser H, Lempert T (2004) Benign paroxysmal positional vertigo predominantly affects the right labyrinth. $J$ Neurol Neurosurg Psychiatry 75: 1487-1488.

11. Epley JM (2001) Human experience with canalith repositioning maneuvers. Ann N Y Acad Sci 942: 179-191. [Crossref]

12. Nuti D, Agus G, Barbieri MT, Passali D (1998) The management of horizontal-canal paroxysmal positional vertigo. Acta Otolaryngol 118: 455-460. [Crossref]

13. Steddin S, Ing D, Brandt T (1996) Horizontal canal benign paroxysmal positioning vertigo (h-BPPV): transition of canalolithiasis to cupulolithiasis. Ann Neurol 40: 918922.

14. Psillas G, Vital I, Rachovitsas D, Vital V, Tsalighopoulos M (2011) Conversion of canalolithiasis to cupulolithiasis in the course of a horizontal benign paroxysmal positional vertigo case. Am J Otolaryngol 32: 174-176. [Crossref]

15. Califano L, Melillo MG, Mazzone S, Vassallo A (2008) Converting apogeotropic into geotropic lateral canalolithiasis by head-pitching manoeuvre in the sitting position. Acta Otorhinolaryngol Ital 28: 287-291.

16. Lempert T, Tiel-Wilck K (1996) A positional maneuver for treatment of horizontalcanal benign positional vertigo. Laryngoscope 106: 476-478. [Crossref]

17. Moriarty B, Rutka J, Hawke M (1992) The incidence and distribution of cupular deposits in the labyrinth. Laryngoscope 102: 56-59. [Crossref]

18. Imai T, Takeda N, Uno A, Horii A, Kitahara T, et al. (2012) Benign paroxysmal positional vertigo showing sequential translations of four types of nystagmus. Auris Nasus Larynx 39: 544-548.

19. Choung YH, Shin YR, Kahng H, Park K, Choi SJ (2006) 'Bow and lean test' to determine the affected ear of horizontal canal benign paroxysmal positional vertigo. Laryngoscope 116: 1776-1781.

20. Kim CH, Kim MB, Ban JH (2014) Persistent geotropic direction-changing positional nystagmus with a null plane: the light cupula. Laryngoscope 124: 15-19. [Crossref]

21. Oas JG (2001) Benign paroxysmal positional vertigo: a clinician's perspective. Ann N Y Acad Sci 942: 201-209. [Crossref]

Copyright: (C2018 Hiruma K. This is an open-access article distributed under the terms of the Creative Commons Attribution License, which permits unrestricted use, distribution, and reproduction in any medium, provided the original author and source are credited. 